\title{
Emergency Medicine Journal
}

\section{Nurse practitioner administered point-of-care ultrasound compared with $x$-ray for children with clinically non- angulated distal forearm fractures in the emergency department: a diagnostic study}

\begin{tabular}{|r|l|}
\hline Journal: & Emergency Medicine Journal \\
\hline Manuscript ID & emermed-2020-209689.R1 \\
\hline Article Type: & Original research \\
\hline Date Submitted by the & n/a \\
\hline Complete List of Authors: & $\begin{array}{l}\text { Snelling, Peter; Griffith University Faculty of Health, School of Medicine; } \\
\text { Gold Coast University Hospital, Emergency Medicine } \\
\text { Jones, Philip; Royal Brisbane and Women's Hospital, Emergency Medicine } \\
\text { Keijzers, Gerben; Gold Coast University Hospital, Emergency Medicine; } \\
\text { Bond University, Professor, Faculty of Health Sciences and Medicine } \\
\text { Bade, David; Queensland Children's Hospital, Orthopaedics } \\
\text { Herd, David; Queensland Children's Hospital, Emergency Medicine; The } \\
\text { University of Queensland School of Medicine, Child Health Research } \\
\text { Centre } \\
\text { Ware, Robert; Griffith University Faculty of Health, Menzies Health } \\
\text { Institute Queensland }\end{array}$ \\
\hline Keywords: & $\begin{array}{l}\text { musculo-skeletal, fractures and dislocations, imaging, ultrasound, } \\
\text { paediatric injury, paediatric emergency med, emergency department }\end{array}$ \\
\hline
\end{tabular}

\section{SCHOLARONE ${ }^{m}$ Manuscripts}


I, the Submitting Author has the right to grant and does grant on behalf of all authors of the Work (as defined in the below author licence), an exclusive licence and/or a non-exclusive licence for contributions from authors who are: i) UK Crown employees; ii) where BMJ has agreed a CC-BY licence shall apply, and/or iii) in accordance with the terms applicable for US Federal Government officers or employees acting as part of their official duties; on a worldwide, perpetual, irrevocable, royalty-free basis to BMJ Publishing Group Ltd ("BMJ") its licensees and where the relevant Journal is co-owned by BMJ to the co-owners of the Journal, to publish the Work in this journal and any other BMJ products and to exploit all rights, as set out in our licence.

The Submitting Author accepts and understands that any supply made under these terms is made by BMJ to the Submitting Author unless you are acting as an employee on behalf of your employer or a postgraduate student of an affiliated institution which is paying any applicable article publishing charge ("APC") for Open Access articles. Where the Submitting Author wishes to make the Work available on an Open Access basis (and intends to pay the relevant APC), the terms of reuse of such Open Access shall be governed by a Creative Commons licence - details of these licences and which Creative Commons licence will apply to this Work are set out in our licence referred to above.

Other than as permitted in any relevant BMJ Author's Self Archiving Policies, I confirm this Work has not been accepted for publication elsewhere, is not being considered for publication elsewhere and does not duplicate material already published. I confirm all authors consent to publication of this Work and authorise the granting of this licence. 


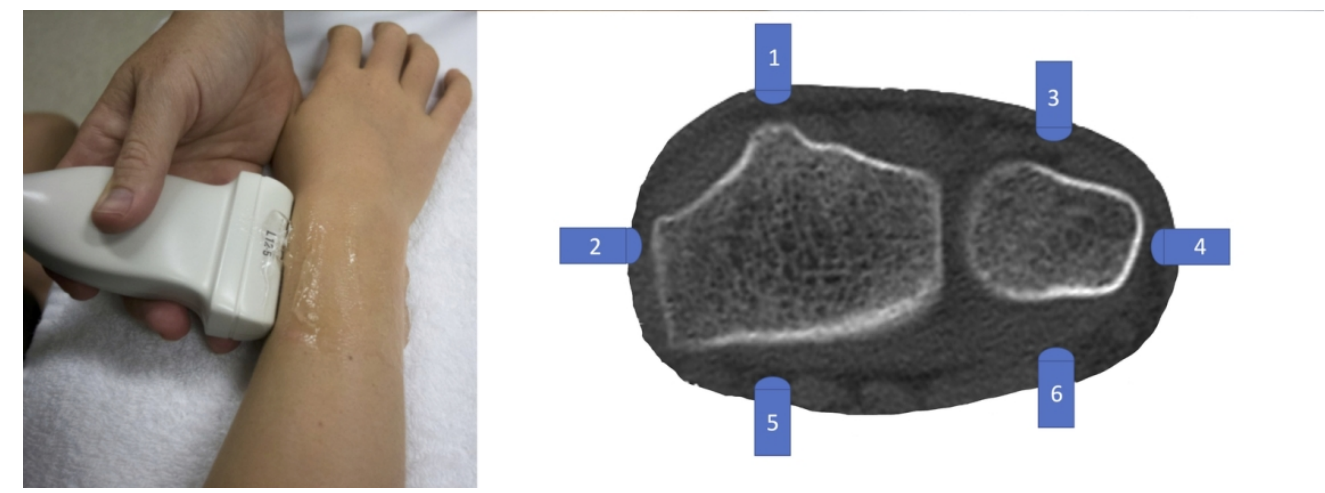

Fig 1. POCUS distal forearm technique and six-view protocol

$$
131 \times 48 \mathrm{~mm}(300 \times 300 \mathrm{DPI})
$$


Figure 2. Participant flow diagram

$262 \times 144 \mathrm{~mm}(72 \times 72$ DPI $)$ 
Figure S1: Time duration to complete POCUS versus $x$-ray imaging $(n=204)$. [Supplementary Figure (online only), no colour requirement]

\section{$176 \times 129 \mathrm{~mm}(600 \times 600 \mathrm{DPI})$}


MS ID\#: emermed-2020-209689

Nurse practitioner administered point-of-care ultrasound compared with x-ray for children with clinically non-angulated distal forearm fractures in the emergency department: a diagnostic study

Peter J. Snelling BSC MBBS(hons) MPHTM CCPU FRACP FACEM, ${ }^{1-5}$ Philip Jones MBBS, ${ }^{6}$ Gerben Keijzers MBBS FACEM PhD, ${ }^{1,3,7}$ David Bade MBBS FRACS FAOrthA, ${ }^{8}$ David W. Herd MBChB FRACP, ${ }^{2,5}$ Robert S. Ware BSc(hons) PhD. ${ }^{1}$

1. School of Medicine and Menzies Health Institute Queensland, Griffith University, Southport, Queensland, Australia

2.Department of Emergency Medicine, Queensland Children's Hospital, South Brisbane, Queensland, Australia

3. Department of Emergency Medicine, Gold Coast University Hospital, Southport, Queensland, Australia

4. Sonography Innovation and Research Group (Sonar Group), Queensland, Australia

5. Child Health Research Centre, University of Queensland, Brisbane, Queensland, Australia

6. Department of Emergency Medicine, Royal Brisbane and Women's Hospital, Queensland, Australia

7. Faculty of Health Sciences and Medicine, Bond University, Gold Coast, QLD, Australia

8. Department of Orthopaedics, Queensland Children's Hospital, South Brisbane, Queensland, Australia

\section{Corresponding author:}

Peter J. Snelling

Department of Emergency Medicine

Gold Coast University Hospital

1 Hospital Blvd

Southport, Qld 4215

E: peter.j.snelling@gmail.com or peter.snelling@health.qld.gov.au

P: 1300774284

Word Count: 3243 


\section{ABSTRACT}

\section{Background}

Paediatric distal forearm fractures are a common emergency department (ED) presentation. They can be diagnosed with point-of-care ultrasound (POCUS) as an alternative to x-rays. Given that ED nurse practitioners (NPs) are relied upon for the diagnosis of paediatric fractures, it is important to describe the diagnostic accuracy of NP-conducted POCUS versus x-ray.

\section{Methods}

This prospective diagnostic study was conducted in a tertiary paediatric hospital in Queensland, Australia. Participants were children aged 4 to 16 years with a non-angulated, suspected distal forearm fracture. Diagnosis from 6-view NP-administered POCUS of the distal radius and ulna was compared against the reference standard of 2-view x-ray. Each patient received both imaging modalities. Overall forearm diagnosis was classified as 'no', 'buckle' or 'other' fracture for both modalities. The primary outcome was diagnostic accuracy for 'any' fracture ('buckle' and 'other' fractures combined). Secondary outcomes included diagnostic accuracy for 'other' fractures versus 'buckle' and 'no' fractures combined, and pain, imaging duration and preference for modality.

\section{Results}

Of 204 recruited patients, 129 had x-ray-diagnosed forearm fractures. The sensitivity and specificity for NP-administered POCUS were 94.6\% (95\% confidence interval [Cl] 89.2-97.3\%) and $85.3 \%(95 \% \mathrm{Cl} 75.6$ to $91.6 \%)$ respectively. 'Other' fractures (mostly cortical breach fractures) when compared to 'buckle'/'no' fractures, had sensitivity $81.0 \%(95 \% \mathrm{Cl} 69.1$ $89.1 \%)$ and specificity $95.9 \%(95 \% \mathrm{Cl} 91.3-98.1 \%)$. Pain and imaging duration were clinically similar between modalities. There was a preference for POCUS by patients, parents, and NPs.

\section{Conclusions}

NP-administered POCUS had clinically acceptable diagnostic accuracy for paediatric patients presenting with non-angulated distal forearm injuries. This included good sensitivity for diagnosis of 'any' fracture and good specificity for diagnosis of cortical breach fractures alone. Given the preference for POCUS, and the lack of difference in pain and duration between modalities, future research should consider functional outcomes comparing POCUS with $\mathrm{x}$ ray in this population in a randomised controlled trial. 


\section{KEY MESSAGES}

\section{What is already known on this subject}

- Point-of-care ultrasound (POCUS) for the diagnosis of paediatric distal forearm fractures against $x$-ray has been demonstrated to be feasible for a variety of healthcare practitioners, including emergency physicians, radiologists and surgeons.

- Nurse practitioners (NPs), who are cost-effective care providers, have not yet been evaluated for this modality.

- Buckle fractures can be managed similarly to a soft tissue injury but have been included as fractures in the diagnostic accuracy of previous studies

\section{What this study adds}

- This study demonstrated that NPs with no prior POCUS experience had acceptable diagnostic accuracy, compared to $x$-ray, when using POCUS to diagnose paediatric, clinically non-angulated, distal forearm fractures

- POCUS accurately ruled-in cortical breach type fractures when compared against buckle fractures and no fractures combined.

\section{INTRODUCTION}

Forearm fractures account for about $1.7 \%$ of children presenting to the emergency department (ED). ${ }^{1}$ They represent about one third of all fractures in children, are mainly distal and usually occur after a fall. ${ }^{2}$ Many are diagnosed as buckle (torus) fractures, which are unique to children due to their malleable cortex within a strong periosteum. ${ }^{3-4}$ Buckle fractures of the distal forearm can be discharged with a wrist splint or soft bandage with no requirement for further imaging or follow-up. ${ }^{4-5}$ The current reference standard to diagnose a distal forearm fracture is 2 -view $x$-ray, ${ }^{6}$ which conveys a small dose of ionising radiation. ${ }^{7}$ Point-of-care ultrasound (POCUS), a portable form of non-ionising imaging conducted at the patient bedside, has been proposed as a potential alternative. ${ }^{8-14}$

Buckle fractures can be conservatively managed akin to soft tissue injuries. ${ }^{5}$ If they can be reliably distinguished from other fractures using POCUS, $x$-rays can potentially be avoided, ${ }^{15}$ which supports the 'as low as reasonably achievable' principle. ${ }^{16,17}$ Furthermore, POCUS performed at the bedside at the time of clinical review may expedite the discharge of patients without a fracture or with a buckle fracture, which could lead to reduced healthcare costs. ${ }^{18}$ Although the use of POCUS for diagnosis of paediatric distal forearm fractures has been shown to be feasible for a variety of healthcare practitioners, including emergency physicians, ${ }^{10-12}$ radiologists ${ }^{13}$ and surgeons, ${ }^{14}$ the ability of nurse practitioners (NPs) to use POCUS for diagnosis of paediatric distal forearm fractures has not been specifically studied. NPs are utilised in the ambulatory care area of the ED, where they provide high-quality, costeffective care with a broad scope of practice. ${ }^{19}$ Additionally, NPs are primary care providers in many rural and remote healthcare facilities. ${ }^{20}$

The aim of this study was to assess the diagnostic accuracy of NPs who had no previous POCUS experience to administer POCUS compared to $\mathrm{x}$-ray in paediatric non-angulated distal forearm fractures. We describe diagnostic accuracy for 'any' fracture ('buckle' and 'other' 
fractures combined), as well as 'other' fractures, comprised primarily of cortical breach fractures, alone (compared with 'buckle' and 'no' fractures combined).

\section{METHODS}

\section{Study design and Setting}

This prospective diagnostic study was conducted at Queensland Children's Hospital (QCH) in Southeast Queensland, Australia between February 2018 and April 2019. QCH is a tertiary paediatric (<16 years age) centre that had approximately 80,000 ED presentations in 2018. The Children's Health Queensland Hospital and Health Service Human Research Ethics Committee approved the study (HREC/17/QRCH/239). Written consent was obtained for all subjects from the patient's legal guardian, hereafter referred to as the parent, prior to enrolment. The study was prospectively registered in the Australian and New Zealand Clinical Trials Registry (ACTRN12617001648314) and has been reported according to STARD guidelines.

\section{Selection of Participants}

Patients were potentially eligible for the study if they were aged 4 to 16 years and presented to the ED within the rostered clinical hours of NPs (7am-10pm). Children under 4 years of age were not included as they were physically too small to fit the wrist splints available at the study hospital. Patients were eligible for inclusion if they were triaged to the ambulatory zone with an isolated, clinically non-angulated distal forearm injury which required further evaluation for a fracture with x-ray imaging. This included soft tissue swelling but not visible deformity. Exclusion criteria were injury older than 1 week at presentation; external imaging had already been performed; known bone disease, such as osteogenesis imperfecta; suspicion of non-accidental injury; congenital bone malformation; open fracture; neurovascular compromise; distracting injury or suspicion for another fracture (e.g. scaphoid or elbow). Demographic characteristics and Australasian Triage Scale scores of patients were documented.

\section{Patient and Public Involvement}

The study was conducted with limited patient involvement. Patients and their caregivers were not included in the design or conduct of the study, although important patient-centred outcomes such as pain and preference were assessed.

\section{Interventions}

All participants received both POCUS and x-ray.

POCUS

All $(n=6)$ participating ultrasound naïve NPs underwent a 2-hour didactic training course, followed by 3 proctored scans on patients, before performing POCUS on eligible patients. The 
training program consisted of a staged learning package with lectures combined with practical training on each other and simulated fracture models. ${ }^{18}$ Patients were clinically examined by NPs prior to any imaging, who recorded their pre-test probability of a cortical breach fracture dichotomously (low versus high) based on their own clinical judgement. A six-view forearm POCUS protocol (Figure 1$)^{21}$ was conducted, whereby the distal radius and ulna were interrogated on their dorsal, lateral and volar aspects with a high frequency linear probe (HFL50xp/15-6MHz, Fujifilm Sonosite Xporte, Bothell, USA) in a longitudinal axis with the probe marker orientated distally. NPs subsequently classified the radius and ulna bones separately as 'no', 'buckle' or 'other' fracture. A 'buckle' fracture was defined by an inward or outward bulge of the bone cortex without cortical breach on any aspect. 'Other' fractures were broadly defined as having a cortical breach, which included greenstick, complete or Salter-Harris (physeal) types. ${ }^{4}$ This category also included bowing fractures and fractures at other forearm sites.

NPs prospectively documented their interpretation of the recorded ultrasound image by labelling it with the diagnosis and saving the image prior to the patient having $\mathrm{x}$-ray imaging. The absence of a label on a set of images was interpreted as 'no' fracture. On the occasions where the NPs were not immediately available to scan eligible consented patients, $x$-ray imaging was obtained first, and NPs subsequently scanned patients, masked to the x-ray. At study completion, all POCUS images were reviewed for correct interpretation by a POCUS expert (PJS), a dual qualified emergency physician and paediatrician with POCUS fellowship training, masked to both the NP diagnosis and $x$-ray.

$X-r a y$

2-view x-ray of the forearm was conducted by radiographers masked to POCUS images and findings. Patients were managed on the basis of the treating clinician's interpretation of the $\mathrm{x}$-ray, in conjunction with the report by a paediatric radiologist once available. X-ray results were reported by a paediatric radiologist, masked to POCUS images and findings. Fractures were classified using the same definitions as for POCUS on the basis of these reports, with independent review of images by a specialist paediatric orthopaedic surgeon for any discrepancies.

\section{Outcome Measures}

The primary outcome was diagnostic accuracy of NP-administered POCUS compared to the reference standard of $x$-ray. For both POCUS and $x$-ray, the overall forearm diagnosis was based upon the most clinically relevant injury identified on the radius or ulna. A case was defined as "true positive" when POCUS and x-ray both diagnosed 'any' fracture type (i.e. 'buckle' and 'other' fractures combined). A "true negative" was defined when 'no' fracture was identified on both POCUS and x-ray. A "false positive" was defined when 'any' fracture was identified on POCUS but 'no' fracture was found on x-ray. A "false negative" occurred when 'no' fracture was found on POCUS but x-ray identified 'any' fracture. Given that buckle fractures can be managed more in keeping as a soft tissue injury, we secondarily compared the 'other' fractures against the 'buckle' and 'no' fractures combined. 
Secondary outcomes included pain score during the imaging (patient-reported and parent observed), duration of imaging completion and preference (patient, parent and NP). To assess pain, both the patient and parent were asked to independently select one of six faces (score $0-10$, intervals of 2 ) that correlated to the maximal pain experienced during both POCUS and x-ray using the Faces Pain Scale Revised (FPSR), a reliable and validated tool for this age range. ${ }^{22}$ Provision and timing of any analgesia were documented. Duration of POCUS was recorded with a digital timer, which included the time taken to locate and turn on the machine, enter patient details and save the final image. Duration of $x$-ray was determined from the electronic medical record, calculated from the time recorded for the order being processed and the time stamp on the final radiograph. X-ray duration was reduced by the time spent performing POCUS, if this occurred between $x$-ray imaging being ordered and performed. Preference for POCUS over $x$-ray was recorded independently by both the patient and parent using a 5-point Likert-type scale. NPs indicated their dichotomous preference for either x-ray or POCUS for that particular patient. FPSR ratings were administered after each imaging modality and preferences were administered at the end of the consultation, prior to discharge. All data were entered prospectively on the case report form by the NPs. Any spurious or missing data were contemporaneously cross checked with research records and with the NP recollection.

\section{Sample Size}

Sample size calculations were based on the assumption that one third of patients would have fractures and that both sensitivity and specificity of POCUS would be $80 \%$. To estimate sensitivity to within $+/-10 \%$ and the specificity to within $+/-7 \%$ we were required to recruit 200 patients. The size of these margins was chosen by the emergency physicians in the study team and determined by what, in their opinion, was a clinically acceptable variation in agreement given the potential advantages of POCUS.

\section{Data Analysis}

Summary statistics were described for continuous data using either mean and standard deviation (SD) or median and interquartile range (IQR) as appropriate, and for categorical data as frequency and percentage. Diagnostic statistics reported include sensitivity, specificity, positive and negative predictive values and positive and negative likelihood ratios, with corresponding 95\% confidence intervals ( $\mathrm{Cls}$ ) calculated using the Wilson method. The agreement between NP POCUS findings and masked expert review were assessed using percentage agreement. Pain scores were compared between-modalities using Student's $t$ test. Imaging duration and patient/parent preference were compared using the Wilcoxon signed rank test. All statistical calculations were performed using Stata/IC v14 (StataCorp, College Station, TX, USA).

\section{RESULTS}

Of 2056 patients who presented with forearm injuries during the study period, 741 were eligible and 204 were recruited (Figure 2). All patients received both POCUS and x-ray imaging. Recruited patients had a mean age of 9.5 years and $52.0 \%$ were male (Table 1 ). 
Eligible patients not recruited were similar in terms of age (mean 9.5 years) and sex $(45.6 \%$ male). The left arm was affected in almost two-thirds of patients. The overall forearm fracture classification was determined by the radius fracture pattern in all cases, apart from one patient with an isolated ulnar styloid fracture. Data was complete for the primary outcome. Data for 1 parent FSPR rating and 3 preference scores (patient and parent) were missing.

Table 1. Patient characteristics, total and per overall forearm diagnosis according to $x$-ray results $(n=204)$

\begin{tabular}{|c|c|c|c|}
\hline Characteristic & $\begin{array}{l}\text { No Fracture } \\
(n=75)\end{array}$ & $\begin{array}{l}\text { Buckle Fracture } \\
(n=71)\end{array}$ & $\begin{array}{l}\text { Other Fracture } \\
(n=58)\end{array}$ \\
\hline Age (y) & $10.1( \pm 3.1)$ & $9.0( \pm 3.0)$ & $9.3( \pm 2.8)$ \\
\hline \multicolumn{4}{|l|}{ Gender } \\
\hline 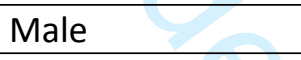 & $33(44)$ & $40(56)$ & $33(58)$ \\
\hline Female & $42(56)$ & $31(44)$ & $25(43)$ \\
\hline \multicolumn{4}{|l|}{ Affected Arm } \\
\hline Right & $31(41)$ & $28(39)$ & $19(33)$ \\
\hline Left & $44(59)$ & $43(61)$ & $39(67)$ \\
\hline \multicolumn{4}{|c|}{ Analgesia Received } \\
\hline No Analgesia & $19(25)$ & $18(25)$ & $6(10)$ \\
\hline $\begin{array}{l}\text { Paracetamol } \\
\text { and/or ibuprofen }\end{array}$ & $55(73)$ & $53(75)$ & $45(78)$ \\
\hline Opioid Analgesia & $1(1)$ & $0(0)$ & $7(12)$ \\
\hline \multicolumn{4}{|l|}{ Triage Category } \\
\hline 3 & $5(7)$ & $7(10)$ & $18(31)$ \\
\hline 4 & $60(80)$ & $54(76)$ & $37(64)$ \\
\hline 5 & $10(13)$ & $10(14)$ & $3(5)$ \\
\hline \multicolumn{4}{|c|}{ Pre-test Clinical Suspicion of Fracture Severity } \\
\hline Low & $66(88)$ & $37(52)$ & $12(21)$ \\
\hline High & $9(12)$ & $34(48)$ & $46(79)$ \\
\hline \multicolumn{4}{|l|}{ POCUS Diagnosis } \\
\hline No fracture & $64(85)$ & $2(3)$ & $5(9)$ \\
\hline Buckle & $8(11)$ & $66(93)$ & $6(10)$ \\
\hline Other fracture & $3(4)$ & $3(4)$ & $47(81)$ \\
\hline
\end{tabular}

There were 129 (63.2\%) patients with at least one fracture detected on x-ray, of which 71 (34.8\%) were diagnosed as 'buckle' fracture (Tables S1 \& S2). POCUS correctly identified 'any' fracture in 122/129 patients with sensitivity $94.6 \%$ (95\% Cl $89.2-97.3 \%$ ) (Table 2). POCUS correctly identified 'no' fracture in 64 of 75 patients, with specificity $85.3 \%$ (95\% Cl $75.6-$ 91.6\%). The overall sensitivity and specificity of POCUS for 'other' fractures alone (versus 'buckle' and 'no' fractures combined) were $81.0 \%$ (95\% Cl $69.1-89.1 \%)$ and $95.9 \%(95 \% \mathrm{Cl}$ $91.3-98.1 \%$ ) respectively (Table 2 ). NP clinical examination pre-test probability was not associated with fracture type (Table 1 ). 


\section{MS ID\#: emermed-2020-209689}

Table 2. Test performance characteristics of POCUS compared to reference standard of $x$-ray for identification of 'any' fracture ('buckle'/'other' fractures combined) and for identification of 'other' fractures ('no'/'buckle' fractures combined)

\begin{tabular}{|c|c|c|c|c|c|c|c|c|c|c|}
\hline $\begin{array}{l}\text { Diagnostic } \\
\text { Study }\end{array}$ & $\mathrm{TP}$ & FN & $\mathrm{TN}$ & $\mathrm{FP}$ & Sensitivity & Specificity & PPV & NPV & PLR & NLR \\
\hline \multicolumn{11}{|c|}{ 'Any' Fracture vs ‘No' Fracture } \\
\hline Radius & 121 & 7 & 66 & 10 & $\begin{array}{l}94.5 \\
(89.1-97.3)\end{array}$ & $\begin{array}{l}86.8 \\
(77.4-92.7)\end{array}$ & $\begin{array}{l}92.4 \\
(86.5-95.8)\end{array}$ & $\begin{array}{l}90.4 \\
(81.5-95.3)\end{array}$ & 7.1 & 0.06 \\
\hline Ulna & 22 & 14 & 161 & 7 & $\begin{array}{l}61.1 \\
(44.9-75.2)\end{array}$ & $\begin{array}{l}95.8 \\
(91.7-98.0)\end{array}$ & $\begin{array}{l}75.9 \\
(57.9-87.8)\end{array}$ & $\begin{array}{l}92.0 \\
(87.0-95.2)\end{array}$ & 14.7 & 0.41 \\
\hline Combined & 122 & 7 & 64 & 11 & $\begin{array}{l}94.6 \\
(89.2-97.3)\end{array}$ & $\begin{array}{l}85.3 \\
(75.6-91.6)\end{array}$ & $\begin{array}{l}91.7 \\
(85.8-95.3)\end{array}$ & $\begin{array}{l}90.1 \\
(81.0-95.1)\end{array}$ & 6.4 & 0.06 \\
\hline \multicolumn{11}{|c|}{ 'Other' Fracture vs 'Buckle' or 'No' Fracture } \\
\hline Radius & 47 & 10 & 141 & 6 & $\begin{array}{l}82.5 \\
(70.6-90.2)\end{array}$ & $\begin{array}{l}95.9 \\
(91.4-98.1)\end{array}$ & $\begin{array}{l}88.7 \\
(77.4-94.7)\end{array}$ & $\begin{array}{l}93.4 \\
(88.2-96.4)\end{array}$ & 20.2 & 0.18 \\
\hline Ulna & 2 & 9 & 192 & 1 & $\begin{array}{l}18.2 \\
(5.1-47.7)\end{array}$ & $\begin{array}{l}99.5 \\
(97.1-99.9)\end{array}$ & $\begin{array}{l}66.7 \\
(20.8-93.9)\end{array}$ & $\begin{array}{l}95.5 \\
(91.7-97.6)\end{array}$ & 35.1 & 0.82 \\
\hline Combined & 47 & 11 & 140 & 6 & $\begin{array}{l}81.0 \\
(69.1-89.1)\end{array}$ & $\begin{array}{l}95.9 \\
(91.3-98.1)\end{array}$ & $\begin{array}{l}88.7 \\
(77.4-94.7)\end{array}$ & $\begin{array}{l}92.7 \\
(87.4-95.9)\end{array}$ & 19.7 & 0.20 \\
\hline
\end{tabular}

TP = True Positive; FN = False Negative; TN = True Negative; FP = False Positive; PPV = positive predictive value; NPV = negative predictive value; PLR = Positive Likelihood Ratio; NLR = Negative Likelihood Ratio

Test Performance reported as \% (95\% Cl calculated with Wilson method) 
Three of the six NPs together performed the majority of POCUS (for $86.8 \%, n=177$ ) of the recruited patients, with all three displaying similar diagnostic accuracy. NP categorisation of POCUS images agreed with masked expert review for $90 \%$ of radius studies (184/204), kappa $=0.79$, and $92 \%$ of ulna studies $(187 / 204)$, kappa $=0.70$. False negatives were only missed by the expert reviewer if saved POCUS images did not contain a view of the fracture. Of 71 patients with buckle fractures on x-ray, 66 were diagnosed correctly with POCUS. Of the remaining 5 patients, 2 were missed by POCUS and 3 were overcalled as cortical breach type fractures. There were 7 patients without a fracture on x-ray diagnosed as buckle fractures with POCUS.

Pain scores were similar for POCUS compared to $x$-ray as recorded by patients (mean difference $-0.10[95 \% \mathrm{Cl}-0.38-0.17]$ ), whilst parents rated x-ray more painful (mean difference 0.35 [ $95 \% \mathrm{Cl} 0.10-0.61$ ]) (Table 3). The median duration of POCUS ( 7 mins [IQR 6 -9]) and x-ray (8 mins [IQR 5.5 - 13.5]) were similar for clinical significance, although delays of greater than 15 minutes occurred more frequently for $x$-ray $(44 / 204,21.6 \%)$ than for POCUS (3/204, 1.5\%) (Figure S1). There was a preference for POCUS by patients, parents (Table S3), and NPs (71.2\%).

Table 3. Differences in pain score, proportions with severe pain and imaging duration for $x-$ ray and POCUS $(n=204)$

\begin{tabular}{|c|c|c|c|c|}
\hline & X-ray & POCUS & Difference $(95 \% \mathrm{Cl})$ & $p$-value \\
\hline \multicolumn{5}{|c|}{ FPSR score - Mean (95\% Cl) } \\
\hline Child & $1.45(1.17-1.72)$ & $1.55(1.28-1.82)$ & $-0.10(-0.38-0.18)$ & 0.47 \\
\hline Parent & $1.55(1.27-1.82)$ & $1.19(0.98-1.41)$ & $0.35(0.10-0.61)$ & 0.007 \\
\hline \multicolumn{5}{|c|}{ Proportion with FPSR score $\geq 6^{*}$} \\
\hline Child & $6.9 \%(14 / 204)$ & $5.9 \%(12 / 204)$ & $1.0 \%(-3.8 \%-5.7 \%)$ & 0.84 \\
\hline Parent & $7.4 \%(15 / 203)$ & $1.5 \%(3 / 203)$ & $5.9 \%(1.9 \%-9.9 \%)$ & 0.006 \\
\hline \multicolumn{5}{|c|}{ Time until Imaging Completed - Median (IQR) ${ }^{\dagger}$} \\
\hline $\begin{array}{l}\text { Time } \\
\text { (Minutes) }\end{array}$ & $8.0(5.5-13.5)^{\ddagger}$ & $7.0(6.0-9.0)$ & $1.7(0.8-2.9)$ & $<0.001$ \\
\hline \multicolumn{5}{|c|}{ Proportion with Image Completion $\geq 15$ minutes ${ }^{*}$} \\
\hline Proportion & $21.6 \%(44 / 204)$ & $1.5 \%(3 / 204)$ & $20.1 \%(14.2 \%-26.0 \%)$ & $<0.001$ \\
\hline \multicolumn{5}{|c|}{$\begin{array}{l}\text { FSPR = Faces Pain Scale - Revised. IQR = Interquartile range; POCUS = point-of-care } \\
\text { ultrasound } \\
\text { Pain scores for POCUS and x-ray each not obtained from } 1 \text { parent. } \\
\text { *Fisher's Exact Test significance level and Wald } 95 \% \text { confidence interval reported } \\
\text { †Related-samples Hodges-Lehman mean difference and confidence interval reported. } \\
\ddagger \text { Adjusted delay to x-ray with POCUS duration subtracted from total time. }\end{array}$} \\
\hline
\end{tabular}




\section{DISCUSSION}

NP-administered POCUS had good sensitivity for 'any' fracture and good specificity for 'other' fractures alone (which were predominately cortical breach fractures) compared to $\mathrm{x}$-ray in patients aged 4 to 16 years presenting to the ED with non-angulated paediatric distal forearm injuries. Patient-reported pain and procedure duration were similar for both imaging strategies. Patients, parents and NPs preferred POCUS over x-ray as an imaging modality.

The diagnostic characteristics of NP-administered POCUS for paediatric distal forearm fractures were comparable to previous studies of POCUS performed by other clinicians within the ED. ${ }^{10-12}$ In contrast to other studies, our study focused on NPs who were true novices to POCUS, with no prior POCUS experience. The benefit of training NPs is their ubiquity as costeffective primary care providers in both urban and rural settings. ${ }^{19}$ Our study affirms that novices can readily obtain proficiency in forearm POCUS after a short training course. Our study extends this previous work by distinguishing 'buckle' fractures from 'other' fractures and pragmatically combined them with the 'no' fracture group for secondary analysis, given that buckle fractures can be managed without manipulation, casting or routine follow-up. The high specificity suggests POCUS a useful rule-in tool for paediatric distal forearm cortical breach type fractures using the described scanning protocol.

The majority of the false negatives in this trial were not deemed clinically significant as per paediatric orthopaedic surgeon review and could be managed conservatively, including a radius bowing fracture, a radial head Salter-Harris 2 fracture, non-displaced ulnar styloid fractures, and non-displaced distal radius greenstick fractures. ${ }^{5}$ However, there were 3 missed fractures that may have been clinically relevant including a greenstick fracture of the distal radius with mild dorsal angulation (not requiring manipulation), a Salter-Harris 2 fracture of the distal radius with mild dorsal angulation (not requiring manipulation), and a proximal third radius complete fracture with mild angulation (receiving manipulation), which was not detected during clinical examination for study eligibility. ${ }^{23}$ All the other 12 angulated fractures that required manipulation were identified on POCUS. In addition to a systematic physical examination, POCUS detection of fractures could be improved by ensuring the entire length of the forearm is scanned and assessing for other signs of fractures, including periosteal hematomas. ${ }^{24}$ The ulna styloid fracture POCUS detection rate may have been increased with closer interrogation of the ulna epiphysis on the lateral view and could be emphasised during training. However, apart from one isolated ulna styloid fracture, all were in association with an equally or more significant detected fracture of the radius, which determined the patient's management.

Most false positives were due to NPs overcalling minor cortical irregularities as buckle fractures, which can be easily resolved by comparison of the same region on the other arm. We hypothesise that POCUS may be more accurate than $\mathrm{x}$-ray in certain circumstances, such as where subtle cortical irregularities or breaches are detected. Some patients with repeat imaging demonstrated callous formation on subtle fractures that were only detected on POCUS imaging. However, this study design did not follow patients longitudinally to specifically assess this. The absence of follow-up meant that we were unable to determine the effect on patient centred outcomes such as wrist function. 
The risk of incorrectly identifying a cortical breach fracture on POCUS could lead to unnecessary immobilisation, which may have resource and convenience implications. The risk of missing a fracture on POCUS could lead to ongoing discomfort and potentially affect bone healing. ${ }^{2}$ Although accepted as standard practice, it is important to note x-ray imaging, and its interpretation, has limitations for fracture detection and can result in both false positives and negatives. ${ }^{25}$ Therefore, using $\mathrm{x}$-ray as the reference standard may underestimate the diagnostic accuracy of POCUS.

Patients reported similar pain scores and parents reported lower pain scores for POCUS versus $x$-ray, in keeping with other literature. ${ }^{10-12}$ However, more patients $(5.9 \%)$ than parents $(1.5 \%)$ reported pain scores $\geq 6$ for POCUS, which may have been due to the anxiety generated by the contact of the probe directly over the fracture site rather than physical pain. Administration of analgesia was similar between groups, apart from opioid analgesia, which was almost exclusively provided for patients with a cortical breach fracture. The timing of analgesia was similar across all patient groups and unlikely to have contributed to any differences in pain ratings.

An additional benefit of POCUS is the potential reduction in the number of $x$-rays performed by screening patients for 'buckle' or 'no' fracture diagnoses. ${ }^{15}$ In our study, $36 \%$ of patients had no fracture and $35 \%$ had buckle fractures, demonstrating an approximate $30 \%$ yield of cortical breach fractures with routinely performed x-rays. ${ }^{17}$ Although the radiation dose for forearm $x$-ray is relatively small, any avoidance of unnecessary ionising radiation in the paediatric population is desirable. ${ }^{7,16}$ However, when a cortical breach is detected on POCUS it cannot always characterise the fracture further, particularly for fractures around the physis, which generally require outpatient follow-up. Therefore, $x$-ray is still required when a cortical breach is detected on POCUS.

A strength of the study was that only patients with non-deformed distal forearm injuries were included in our study, given that fractures would otherwise be clinically apparent and would artificially inflate the test characteristics of POCUS. Interestingly, 13 fractures were manipulated in this cohort of patients, indicating that angulation can be masked by swelling. Reporting of our primary outcome, the diagnostic accuracy for the detection of any fracture, is consistent with previous research. As an additional strength, we also reported the diagnostic accuracy of the 'other' fracture group alone (primarily consisting of cortical breach fractures at the distal radius), which is more consistent with clinical practice.

There were several limitations to this study. This was a single site study conducted at a tertiary paediatric centre and, as such, findings may not be directly applicable to other settings. Given the pragmatic nature of this study, patients were recruited as a convenience sample within NP rostered hours with the potential for recruitment or sampling bias. The 535 eligible patients who were not recruited had similar baseline demographics, which makes systemic bias unlikely. NPs were not blinded to the history and could examine patients prior to or during POCUS. Although this reflects real-world medicine, it may have increased the accuracy of POCUS compared to $\mathrm{x}$-ray. X-ray was used as the reference standard in this study and reflects current routine ED practice. However, $x$-ray is not the gold standard and may have underestimated the true diagnostic accuracy of POCUS. X-rays were obtained prior to POCUS 
in a minority of cases, which may have affected secondary outcomes. Although there was no clinically significant difference in the median duration of imaging, we did not measure total ED length of stay.

\section{Conclusion}

In this study, NP-administered POCUS had acceptable diagnostic accuracy for paediatric patients presenting to the ED with non-angulated distal forearm injuries. We found a good sensitivity for all fracture types combined (buckle fractures included) and good specificity for the more serious cortical breach fractures. POCUS has the potential to safely rule-out a fracture or rule-in a cortical breach fracture and expedite further management. Given that POCUS is not more painful than x-ray and is acceptable to patients, parents and NPs, future research should assess medium-term patient centred outcomes of POCUS compared to x-ray and investigate the cost effectiveness of both modalities.

\section{Author contributions:}

PJS conceived the study and designed the trial. DB, DWH and RSW contributed to the study design. PJS and DWH obtained research funding. PJS supervised the conduct of the trial and data collection. PJS undertook recruitment of participating centre and patients and managed the data, including quality control. PJ, GK and RSW provided statistical advice on study design and analysed the data. PJS drafted the manuscript, and PJ, GK and RSW contributed substantially to its revision. DB and DWH reviewed the final manuscript. PJS takes responsibility for the paper as a whole

\section{Acknowledgements:}

Mark Moore for assisting with recruitment and data audit, Peta Gimpel for assisting with recruitment and data entry and Rosemary Rogers for assisting with recruitment and data entry.

\section{Conflicts of interest:}

None to declare.

\section{Funding:}

Study, Education and Research Trust Account (SERTA) Research Grant, Round 2, 2017, Children's Health Queensland, Australia. The funding body had no role in the conduct or reporting of the research.

\section{Meetings:}

Presented by PJS as an oration at the World Federation for Ultrasound in Medicine and Biology (WFUMB) International Conference Sep 2019, Melbourne, Victoria, Australia. 
MS ID\#: emermed-2020-209689

\section{REFERENCES}

[1] Acworth J, Babl F, Borland M, et al. Patterns of presentation to the Australian and New Zealand Paediatric Emergency Research Network. Emerg Med Australas. 2009; 21:59-66.

[2] Rodriguez-Merchan EC. Pediatric fractures of the forearm. Clin Orthop Relat Res. 2005; 432: $65-72$

[3] Currey J, Butler G. The mechanical properties of bone tissue in children. J Bone Joint Surg. 1975. 57(6):810-14.

[4] Little JT, Klionsky NB, Chaturvedi A, et al. Pediatric distal forearm and wrist injury: an imaging review. Radiographics, 2014; 34:472-490.

[5] Handoll HH, Elliott J, Iheozor-Ejiofor Z, et al. Interventions for treating wrist fractures in children. Cochrane Database Syst Rev. 2018; 12. No.:CD012470.

[6] Metz VM, Gilula LA. Imaging techniques for distal radius fractures and related injuries. Orthop Clin N Am. 1993; 24:217-228.

[7] Brenner DJ, Doll R, Goodhead DT, et al. Cancer risks attributable to low doses of ionizing radiation: assessing what we really know. Proc Natl Acad Sci U S A.

2003;100(24):13761Y13766.

[8] Douma-den Hamer D, Blanker MH, Edens MA, et al. Ultrasound for distal forearm fracture: A systematic review and diagnostic meta-analysis. PLOS ONE. 2016; 11(5):e0155659.

[9] Chartier LB, Bosco L, Lapointe-Shaw L, et al. Use of point-of-care ultrasound in long bone fractures: a systematic review and meta-analysis. CJEM. 2017; 19:131-42.

[10] Poonai N, Myslik F, Joubert G, et al. Point-of-care ultrasound for non-angulated distal forearm fractures in children: test performance characteristics and patient-centred outcomes. Acad Emerg Med. 2017; 24(5):607-616. doi: 10.1111/acem.13146. Epub 2017 Apr 27.

[11] Rowlands R, Rippey J, Tie S, et al. Bedside ultrasound vs x-ray for the diagnosis of forearm fractures in children. Int J Emerg. 2017; 52(2):208-215.

[12] Epema AC, Spanjer MJB, Ras L, et al. Point-of-care ultrasound compared with conventional radiographic evaluation in children with suspected distal forearm fractures in the Netherlands: a diagnostic accuracy study. Emerg Med J. 2019; 36:613-616.

[13] Williamson D, Watura R, Cobby M. Ultrasound imaging of forearm fractures in children: a viable alternative? J Accid Emerg Med, 2000; 17:22-24 
[14] Hubner U, Schlicht W, Outzen S, et al. Ultrasound in the diagnosis of fractures in children. J Bone Joint Surg Br. 2000;82(8):1170-1173.

[15] Snelling PJ. Getting started in paediatric emergency medicine point-of-care ultrasound: Five fundamental applications. Australasian Journal of Ultrasound in Medicine. 2020; 23(1):5-9.

[16] Cohen MD. ALARA, image gently and CT-induced cancer. Pediatr Radiol. 2015 Apr;45(4):465-70. doi: 10.1007/s00247-014-3198-3. Epub 2015 Feb 14.

[17] Alzen G, Duque-Reina D, Urhahn R, et al. Radiographic examination of injuries in children: clinical and legal considerations about indications. Dtsch med Wochenschr. 1992;117:363-7.

[18] Snelling PJ. A Low-cost ultrasound model for simulation of paediatric distal forearm fractures. Australasian Journal of Ultrasound in Medicine. May 2018; 21(2):70-74. https://doi.org/10.1002/ajum.12083

[19] Carter AJE, Chochinov AH. A systematic review of the impact of nurse practitioners on cost, quality of care, satisfaction and wait times in the emergency department. CJEM. 2007; 9(4):286-95.

[20] MacLellan L, Higgins I, Levett-Jones T. Medical acceptance of the nurse practitioner role in Australia: A decade on. J Am Assoc Nurse Pract. 2015;27:152-159.

[21] Ackerman $O$, Liedgens $P$, Eckert $K$, et al. Ultrasound diagnosis of juvenile forearm fractures. J Med Ultrasonics. 2010; DOI 10.1007/s10396-010-0263-x

[22] Hicks CL, Baeyer von CL, Spafford PA, et al. The Faces Pain Scale-Revised: toward a common metric in paediatric pain measurement. Pain. 2001 Aug 1;93(2):173-83.

[23] Larsen E, Vittas D, Torp-Pedersen S. Remodeling of angulated distal forearm fractures in children. Clin Orthop Relat Res. 1988; 237:190-195.

[24] Wang CC, Linden KL, Otero HJ. Sonographic evaluation of fractures in children. JDMS. 2017; 33(3):200-207.

[25] Mounts J, Clingenpeel J, McGuire E, et al. Most frequently missed fractures in the emergency department. Clinical Pediatrics. 2011; 50(3):183-186. 
MS ID\#: emermed-2020-209689

\section{SUPPLEMENTARY TABLES}

Table S1. Fracture types diagnosed by $\mathrm{x}$-ray for patients $(n=129)$

\begin{tabular}{clll} 
Radius & \multicolumn{3}{c}{ Ulna } \\
Buckle & 71 & Buckle & 25 \\
Greenstick & 23 & $\begin{array}{l}\text { Transverse } \\
\text { Styloid }\end{array}$ & 1 \\
Transverse & & & 10 \\
Distal & 7 & & \\
$\begin{array}{c}\text { Proximal } \\
\text { Salter-Harris }\end{array}$ & 1 & & \\
Distal & 24 & & \\
Proximal & 1 & & \\
Bowing & 1 & &
\end{tabular}

Table S2. Overall forearm fracture categories diagnosed by x-ray and POCUS.

\begin{tabular}{|l|c|c|c|c|}
\hline & \multicolumn{2}{|l|}{ X-ray diagnosis } & \\
\hline POCUS diagnosis & 'Other' & 'Buckle' & 'No' & Total \\
\hline 'Other' & 47 & 3 & 3 & 53 \\
\hline 'Buckle' & 6 & 66 & 8 & 80 \\
\hline 'No' & 5 & 2 & 64 & 71 \\
\hline Total & 58 & 71 & 75 & 204 \\
\hline
\end{tabular}

Table S3. Patient and parent responses for preference of imaging modality between POCUS and $\mathrm{x}$-ray $(n=201)$. [Supplementary table online]

\begin{tabular}{|l|l|l|l|l|l|l|}
\hline & \multicolumn{5}{|l|}{ Likert Scale* Response Frequency $^{*}$} & \\
\hline & Strongly Agree & Agree & Neither & Disagree & $\begin{array}{l}\text { Strongly } \\
\text { Disagree }\end{array}$ & -value $^{+}$ \\
\hline Patient & 21 & 74 & 67 & 34 & 5 & $<\mathbf{0 . 0 0 0 1}$ \\
\hline Parent & 34 & 91 & 65 & 10 & 1 & $<\mathbf{0 . 0 0 0 1}$ \\
\hline
\end{tabular}

*"You prefer the use of ultrasound compared with $x$-ray imaging for the detection of your forearm fracture" for patient and "You prefer the use of ultrasound compared with x-ray imaging for the detection of your child's forearm fracture" for parent.

tp-value for null hypothesis of no preference for ultrasound or x-ray using the Wilcoxon matched-pairs signed-ranks test. 